lining was of from two to eight layers of transitional epithelium, the superficial layer being cylindrical. This was a cyst of the duct.

CASE 12 (Gyn. Path., 4535).-Age 35. Cyst $4.5 \times 3 \times 3 \mathrm{~cm}$. egg shaped, thin walled, transparent, and showing on the inner surface several small, shallow, sickle-like openings of the dilated secondary ducts. The cyst wall was of fibrous tissue; the gland elements were mostly normal, a few dilated. The cyst lining, in some places, consisted of one layer of high, pale staining cells, side by side, transitional epithelium from 2 to 6 $r$ more layers thick. Unfolding of ducts and glands.

Case 13 (Gyn. Path., 4643).-Age 23. Size $7 \mathrm{~mm}$. The cyst was situated in the left labium and was very hard.

Case 14 (Gyn. Path., 5019).-Age 39. Cyst $3 \mathrm{~cm}$. in diameter. The cyst wall was of fibrous tissue, and the cyst lining was composed of one layer of cylindrical epithelium.

CASE 15 (Gyn. Path., 5174).-Age 34. Cyst small. The cyst wall was of fibrous tissue, and the cyst lining was made up of one layer of columnar epithelium, with the nuclei at the base of the cells.

CASE 16 (Gyn. Path., 5250).-Age 17. The cyst wall was of fibrous tissue and the cyst lining was composed of several layers of transitional epithelium; at some points there was one layer only.

CAsE 17 (Gyn. Path., 5509).-Age 25. Cysts very small, multiple. The cyst wall was mostly of gland tissue; there were a few cysts varying from a pin point to $2 \mathrm{~mm}$. in diameter. The cysts were mostly dilated secondary ducts, lined in part by transitional epithelium, and in part by one layer of cylindrical epithelium.

\section{SOME NEW VIEWS RESPECTING THE HOROPTER.*}

GEORGE T. STEVENS, Pir.D., M.D.

\section{NEW YORK.}

No question in physiologic optics has been more attractive to investigators in that department of research than that of the horopter, yet, although the ablest thinkers have devoted themselves to it with great earnestness. the subject has remained an unsolved enigma. That the doctrines and theories which have been advanced have not solved the problem, no one who has studied it can doubt. The most notable of all these doctrines, that of the great master Helmholtz, is as unsatisfying, if not more unintelligible, than the others. So far as his marvellous array of abstruse mathematical formulæ served to indicate even a single horopter it was an impossible one both in form and position.

If we inquire into the cause of the failure to solve this interesting and, as I believe, this most important problem. we shall find that it is due to a misunderstanding of the two fundamental principles on which any doctrine of horopters must be formed.

These two principles are: 1 . The doctrine of corresponding points. 2. The doctrine of the directions of the meridians of the retinas.

The first of these principles has been stated incorrectly by all investigators alike, while the second has been incorrectly affirmed by Helmholtz, and those who have accepted his conclusions drawn simply from his own peculiarities.

We have, then, first of all to examine these two fundamental principles.

The doctrine of corresponding points as stated by Helmholtz, who expresses the generally accepted view. is as follows:

* Read In the Section on Ophthalmology of the American Med ical Association. at the Fifty-fifth Annual Session. June. 1004.
1. "On apparent vertical concordant lines, points which are at the same distance from the vertical horizon are concordant."

2. "Points, which in the retinal horizons are at equal distances from a point of fixation, are corresponding points."

Le Conte expresses the law by saying that if a pair of dividers is opened until they are the exact distance apart of the central spots (maculæ) and, while being held level, touch many points, all these points are corresponding points.

Helmholtz proceeds to show that the corresponding points in the two visual fields are also at the same height and separated by the same angle. He illustrates his proposition by stereoscopic and other diagrams which are drawn on a flat surface.

It requires but a moment of consideration to discover that there is here a marked inconsistency.

If points equally distant in the plane of the visual field, as represented by these flat surfaces, are corresponding, as Helmholtz asserts and proves, then it is impossible that points equally distant in the retinal fields should also be correspondent.

Every proof that Helmholtz brings to sustain his interpretation of the law of corresponding points is positive evidence of the incorrectness of his view and the view which has been universally accepted.

If then. the accepted view is incorrect, what is the correct statement of the law? Accepting the law that points in the plane of the visual field, which are at equal distances and separated by equal angles are corresponding points in the visual field, it is at once apparent that the retinal corresponding points must be variable in distance depending on their relative distances from the points of fixation, the muculæ.

I have elsewhere defined corresponding points of the retinas as "those points in the retinas which answer to proportional degrees of rotations of the eyes about the centers of rotation." They represent, therefore, the relation between the muscular and the retinal senses.

The belief that for retinal spatial locations equal distances in space must be impressed on equally distant points of the retina is an entirely false conception of the functions of that organ.

That some recent observations have appeared to show that estimation of space may be, within very limited extents, made without corresponding movements of the eyes, does not invalidate the proposition that the corresponding points of the retinas represent the relation between the muscular and retinal senses. We shall presently see that another element enters into the ideas of space which emphasizes the psychologic side of the question to the modification of the purely mechanical side.

The second fundamental principle in the question of the horopter is the position of the meridians of the retina relative to the cranium.

Helmholtz, examining by a method not fully reliable, found that, as he believed. the vertical meridians of the retinas leaned outward, while the horizontal meridians remained exactly horizontal. This does not permit of a discussion of this proposition or the reason for these apparent peculiarities. I have elsewhere shown the probable reason for this error. It is sufficient to say that the conditions of arjustment of the great philosopher's eyes, which he has incidentally revealed, confirm the belief that his meridians leaned out, but there is no reason to suppose that his observations on his own horizontal meridians were correct. There is every reason to believe that they were erroneous. 
The leaning out of his vertical meridians did not, as he assumed, represent the typical adjustment of eyes; they were, in fact, rather exaggerated instances of an anomalous state.

The clinoscope has cleared this subject of all mystery and shows that there are various anomalies of the positions of the vertical-and, therefore, of all-meridians just as there are various anomalies of refraction. But the typical position for the vertical meridians is precisely vertical.

Now, assuming the law of corresponding points as I have stated it, and assuming the verticality of the vertical meridians, the necessity for all the involved and almost endless mathematical formulæ of Helmholtz in determining a horopter is eliminated and these intricate

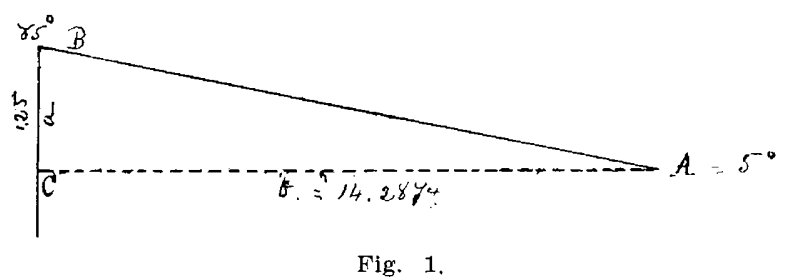

formulæ may be replaced by simple problems of plane trigonometry.

The horopters of former investigators have been based on a doctrine of corresponding points of equal measurements on the surface of the retina. The horopter, according to the present reasoning, is based on corresponding angular displacements which vary in the two eyes in
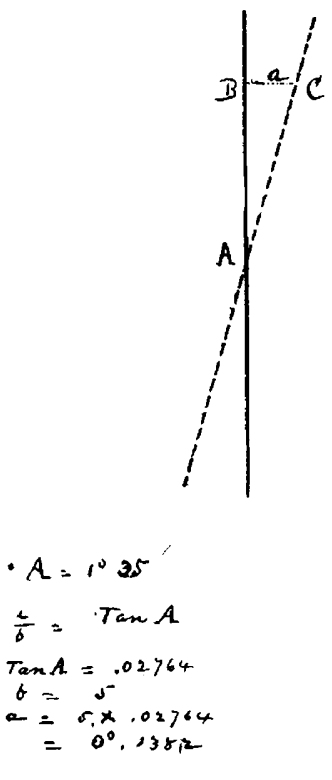

Fig. 2.

proportion as a given object in the visual field is removed to one side of the point of fixation.

With the data at our command derived from these two basal principles, we may then compute a horopter when the eyes are directed straight forward to a point at infinite distance, the head being in the primary position, but for other positions further data are demanded. In convergence, and with depression of the plane of vision, the phenomena arising from the laws of torsions must enter into our computations.

For example, if the two eyes are in convergence and the plane of regard is depressed the horizontal meridians of each eye will tilt downward toward the temporal side, and the upper end of the vertical meridians will tilt out while the lower ends will tilt in. These tiltings in typically adjusted eyes are accurately proportioned to the lateral and ascensional displacements of the eves. Here permit me to diverge from my topic long enough to allude to the absurd custom of some writers who confound the two absolutely different and independent conditions, torsions and declinations, the first physiologic rotations, the other anomalous adjustments.

Employing all these data we are then in position to determine the general positions of a horopter for different adjustments.

In the case we have already supposed, with the eyes directer to the horizon and at infinite distance, the

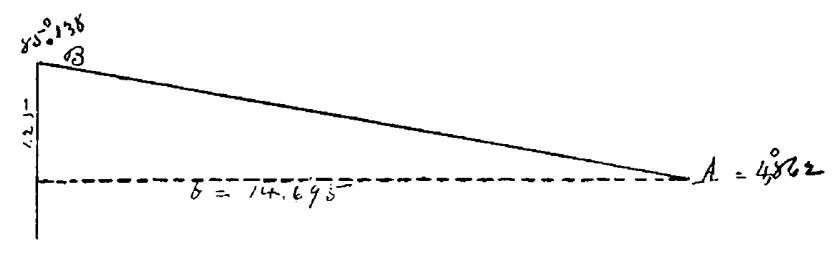

Fig. 3.

horopter will be formed in relation with the distant point of fixation and will be a plane surface at a right angle to the plane of regard. If we were to accept only a purely mechanical interpretation of visual phenomena, in this case all objects nearer than the point of fixation would be excluded from the horopter. As a matter of fact there is a subjective horopter, formed on the basis of such unconscious conclusions as enter into the experiment of Dove, where distinctly heteronymous images are seen not only single but correctly located as to distance.

The subjective horopter is of the utmost importance, and is governed by fixed laws. The physical horopter, including the points in space actually impressed at corresponding points of the two retinas may be regarded as the skeleton, while the subjective horopter, including points in space which are impressed at such points on the retinas as correspond to a demand for certain exact movements of the eyes necessary in order to place the image at the macula of each eye constitute a mental assemblage of the lineaments of harmonic visual impressions. In a rude way we may compare this subjective horopter to the overtones of a musical sound. The fun-

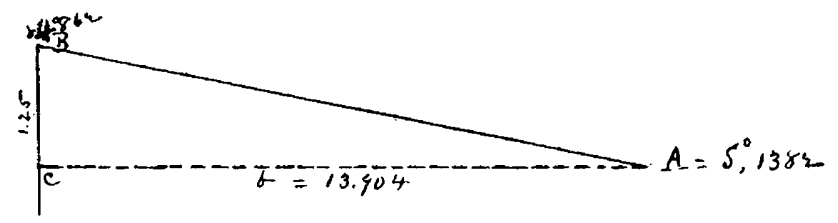

Fig. 4.

damental tone by itself is not pleasing; it is noise. It is the skeleton of the tone. But when combined with its multiplicity of overtones, tones which even the ear of the expert musician fails to differentiate, the musical tone finds its body and affords pleasure to the listener.

In the subjective horopter the consciousness of the required movements takes the place of the actual movement as it does in the Dore experiment.

This doctrine of a subjective horopter does not by any means provide for a confused jumble of space perceptions either in the retinas or in the mind. Every object in the subjective horopter is as much subject to the 
laws of the angular valuation of muscular movements as are the objects in the physical horopter. Keep in mind the principle that while the image of a given point in space may fall on physically non-corresponding points of the retinas, a subliminal consciousness determines the relative positions of these two non-corresponding points to the maculæ of the two eyes and concludes on the movements which would be demanded to make an actual adjustment for the maculæ were such movements to be executed, and it is this unconscious conclusion, based on accurate data, that places the object, singly, in its appropriate position in the field of view and at the proper distance relative to other objects in that field.

In our horopter, objects less distant than the point of fixation may still be within this subjective horopter, their images being impressed on the two retinas at corresponding points for the lines of incidence on one or other side of the maculæ. Then they will appear, as in the spark in the Dove experiment, as single. The principles controlling the psychic phenomena in that experiment are, in this case, modifled to meet the different conditions. Thus, a great number of objects within a fairly wide range may be brought within a perfect horopter.

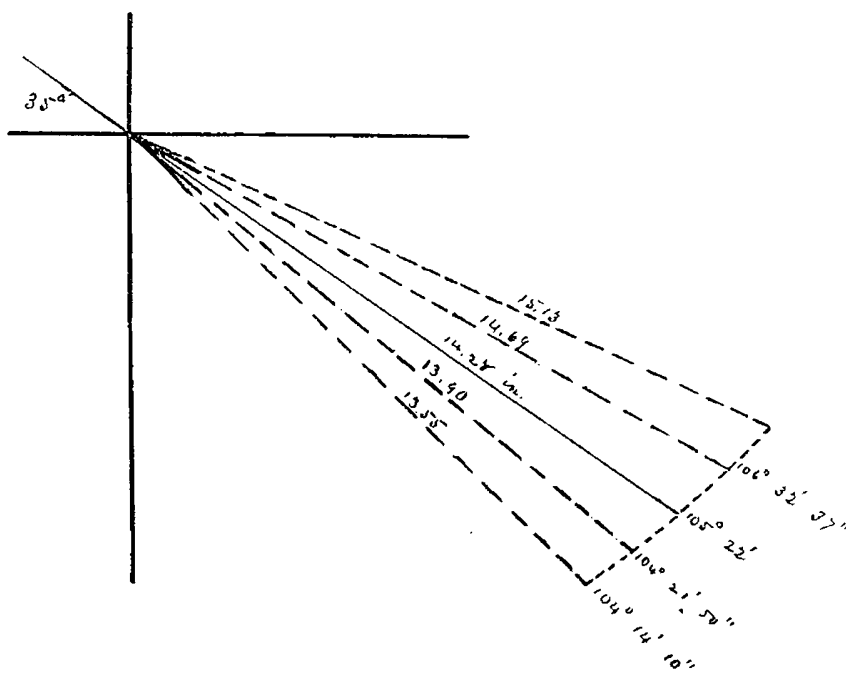

Fig. 5 .

If the direction of the gaze is changed while the head remains in the primary position a new horopter must be and is formed.

Let the gaze be directed downward and to a point a few feet distant. The torsional element is so slight that the former calculations will very nearly give the position of the horopter. Should, however, the gaze be fixed on an object, as for example, the page of a book held in the ordinary position of reading, say, with the point of fixation in the median plane, the plane of regard depressed 35 degrees, and with the lateral deviation of the visual lines (the convergence) for each eye 5 degrees, while the distance between the nodal points is assumed to be $21 / 2$ inches, the problem becomes more complicated. We may determine, from these data, the distance of the horopter and, taking the torsions into consideration, the position of that horopter relative to the plane of regard.

The distance of the point of fixation from the base connecting the two nodal points is determined by the formula, $\mathrm{b} / \mathrm{a}=\operatorname{Cot} \mathrm{A}$, in which $\mathrm{A}=5^{\circ}$ (the lateral angle), $a=1.25$ ( $1 / 2$ the base line) and $b$ the distance required (Fig. 1).
This distance is found to be $14.28 \% 5$ inches, and at this distance the image of the point of fixation is exactly at the macula of each eye.

Images of points in the horizontal line passing through the point of fixation on the page require special consideration here, and there enters at once an apparent difficulty, for the horizontal meridians of the two retinas are no longer horizontal by the law of torsions. If we consult Helmholtz's table of torsions we find that the horizontal (and vertical) meridians have, by a convergence of $5^{\circ}$ in each eye and a depression of the gaze of $35^{\circ}$, been tilted, the horizontal with the outer part down and the inner part up to the extent of $1^{\circ} 35^{\prime}$.

Is it possible now for the images of points of a horizontal line on the page to be impressed at the horizontal meridians of the two retinas, seeing that the former is exactly horizontal and the latter are tilted?

It is not only possible, but in fact exactly because of this tilting of the retinal meridians, that the images of points in the horizontal line of the visual field will be impressed precisely at the horizontal meridians of the retinas.

Without giving space to the mathematical demonstration of this statement, we pass to inquire in what position will lie a horopteric line running up the page and through the point of fixation.

Let us select a point of $5^{\circ}$ above and another $5^{\circ}$ below the point of fixation for the determination of the direction of this ascending line.

It has been shown that the point of fixation is distant from the base line 14.2875 inches. Will the distances to the points $5^{\circ}$ above and $5^{\circ}$ below be the same? Evidently not, for, in order that the image of the upper point on the page shall be impressed at the vertical meridians, the angle of incidence must be less, since owing to the tilting of the vertical meridians they are less widely separated below the maculæ (where the upper image is impressed) than at the maculæ; and because the vertical meridians separate above the maculæ the angle of incidence from the lower point will be greater.

Taking these facts with the given data, we find first the actual extent of the spreading at a point $5^{\circ}$ above the macula. This is determined by the formula, $\mathrm{a} / \mathrm{b}=\operatorname{Tan} \mathrm{A}$, in which $\mathrm{A}=1^{\circ} 35^{\prime}, \mathrm{b}=5^{\circ}$ and $a$ is the distance through which the point $\mathrm{B}$ has been moved to $\mathrm{C}$ (Fig. 2) by the act of torsion.

The extent of change of position outward of this point then is, by the formula, $0^{\circ}, 1382$. Hęnce, in determining the distance of the upper selected point we must subtract from the lateral angle of $5^{\circ}$ the extent of the removal of the point $B$, or $0^{\circ}, 1382$. Also for determination of the distance of the lower selected point, we must add, $0^{\circ}, 1382$ to the $5^{\circ}$ (Fig. 3 ). Then by the first formula, $\mathrm{b} / \mathrm{a}=\operatorname{Cot} \mathrm{A}$, in which $\mathrm{A}=4^{\circ}, 862$, or $5^{\circ}, 1382$, we obtain the distances 14.695 inches for the upper selected point, and 13.904 inches for the lower. Connecting these through the point of fixation we have a line representing the horopteric line running up the page of the book which will form an angle above the line from the fixation point of 105 , which represents also the angle at which the book should be held in order to form a perfect hornpter under the conditions given and with typically adjusted eyes (Fig. 4).

If these calculations are carried further, it is found that in the space of $10^{\circ}$ up and down the page there is a very slight curve, as is shown by the diagram (Fig 5 ).

Experimental research has fully confirmed the results of these calculations. 
The principles which have been shown in these calculations may be applied to any other line in the field of what may be called the objective horopter, while the innate conception of the comparative relations of objects not in this objective horopter serves for the formation of the subjective horopter.

A horopter will be formed in relation to every point of fixation and with as strong, if not with a much stronger, impulse than that of accommodation. Difficulties will be overcome when possible, at the expense of much effort and the habitual failure to make such adjustments of the cyes as to form a fairly complete horopter is attended by unpleasant and even serious results.

The horopter then, is not the over-refinement of scientific curiosity. It is a practical, even momentous subject worthy of the most earnest consideration.

In what has been said it has been assumed that the adjustments of the eyes are typical. Unfortunately such adjustments are not only not universal, but anomalous conditions of adjustments are of great frequency.

Such anomalies may serve as slight hindrances in the formation of a horopter or they may absolutely prevent it. In either case, the instinct to conquer the difficulty is present and efforts are made to overcome the obstacles.

In order to illustrate this proposition let us take our supposed case of one reading with a convergence of the eves of $5^{\circ}$ each, with a depression of the plane of regard of $35^{\circ}$. If there is perfect adjustment of the eyes the horopter is formed as we have shown.

Suppose, however, that the person thus engaged with the book has an adjustment of the eyes of $10^{\circ}$ of arc higher than the typical adjustment; such cases are not rare, they are, on the contrary, very common. Consider what must occur. This person must depress the plane of regard, not $35^{\circ}$, but $45^{\circ}$. But with a convergence of $5^{\circ}$ each eye, and a depression of $45^{\circ}$ the torsion is no longer $1^{\circ} 35^{\prime}$, but much more, therefore no horopter will be formed if the head of this reader remains in the same position as that of the person at first supposed. Two contingencies present themselves, the field of view may remain confused or the reader may advance the head $10^{\circ}$. This he prefers to do, and by this means a compromise is made by which a fairly good horopter is obtained. The same person advances the head also in walking or in conversation.

But suppose again that this person has the eyes adjusted too low by $10^{\circ}$; in such a case no horopter can be formed at the proper position. It can be done by throwing the head backward. We see these people with their chins in the air everywhere.

Again, the reader may have anomalous declinations, and here an obstacle to the formation of the horopter is interposed and the anomalous adjustments just referred to may be and often are complicated with anomalous declinations.

Are these and other maladjustments simple inconveniences? They are, on the contrary, conditions of great moment.

Tet us recall the person with the eyes adjusted too high and who throws the head forward in order to form a horopter. It is in this class that we find the consumptive. It is no accidental coincidence. The consumptive is, in almost all instances, a person who has by the malposition of the air passages induced by the automatic efforts to form the horopter, restricted the circulation of air in the upper bronchi and has thereby offered a hospitable welcome to the bacillus of consumption. I speak from experience when I say that if before the ravages of the parasite have become too extensive the pose of the head is favorably modified by a change in the ocular adjustments the introduction of full currents of fresh air into the upper part of the lungs will banish the intruder and save the victim.

So, too, we might point to the numerous ills induced by the opposite malposition of the hear, but I need not dwell on this. It is proper to add, however, that certain forms of declination will induce these undesirable head positions as well as the high or low adjustments of the eyes.

These are conditions to which I have more than once referred, but it will not be too often till greater importance is attached to the statements.

A word about strain. The results of these atypical conditions are not so much, as nearly all writers about them seem to think, due to strain. For example, it is not the strain on the inferior recti in the case of the too high adjustment. It is the confusion from the excess of torsion which induces a perplexing effort of all the muscles of the eyes as well as a modification of the pose of the head. It would serve the cause of correct thinking if the phrase "eye strain" were completely eliminated from our vocabularies.

I have endeavored to condense into an exceedingly brief space one of the most important subjects with which our ophthalmologist can occupy his attention. If the setting forth has been incomplcte I trust that I may at least have created interest in the subject.

\section{DISCUSSION.}

Dr. G. C. Savage, Nashville, Tenn.-I will be able to prove, by means of this illustration, that corresponding retinal points are points similarly related to the two maculas, not by linear measurement but in degrees of arc. All are agreed that with rare exceptions the macula of one eye corresponds with the macula of the other eye. I had thought that we all likewise agreed that Helmholtz was right when he taught that all other corresponding retinal points bear a similar relationship to the maculas. If Helmholtz had correctly constructed his horopter he would have had at hand a mathematical proof of the correctness of his statement. The true horopter, the isogonal circle, or the monoscopter, is constructed through the point of fixation and through the centers of rotation of the two eyes. These three points, as shown in the illustration, are $c, b$ and $d$, and the circle itself is $c-a-b-d-e$. Every point on this circle

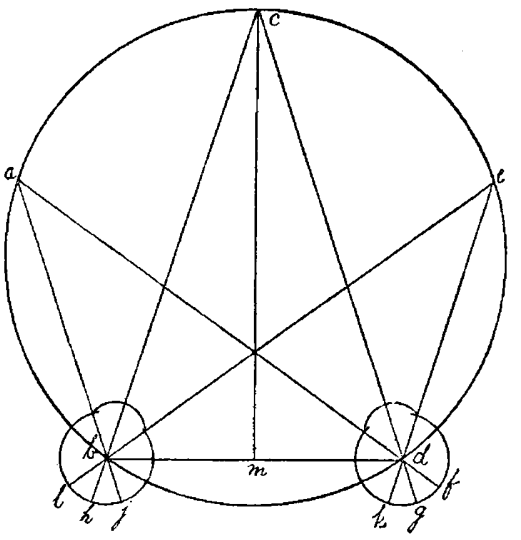

capable of throwing light into the two eyes, will be seen singly by the two eyes, for the images will fall on retinal points that correspond. In the illustration the visual axes $h-c$ and $g-0$ and the indirect visual lines $j-a, f-a, l-e$ and $k \cdot e$ all lie in the plane of the circle $a-b-d-e-c$. It is evident that this plane prolonged backward would include the horizontal retinal meridian of each eye. The are $l-j$ is a part of the one horizontal retinal meridian and the arc $k-f$ is a part of the other horizontal retinal meridian. The macula in one eye is at $h$ and in the other eye is at $g$. The macula $h$ corresponds with macula $g$, 
for the visual axis $h-b-c$ intersects the visual axis $g-d-c$ at $c$, therefore point $c$ is one point for the two eyes. Point $l$ in the one eye corresponds with point $k$ in the other eye, for the visual line $l-b \cdot e$ intersects the visual line $k-d-e$ at $e$, therefore point $e$ is one for the two eyes. Since $l$ and $k$ are corresponding retinal points it only remains for me to prove that $l$ bears the same relationship to $h$ that $k$ does to $g$. The inscribed angle $c \cdot b \cdot e$ is equal to the inscribed angle $c-d-e$, for each is measured by half the are $c-e$. The angle $l-b-h$ is equal to the angle $c-b-e$, for they are opposite angles; and the angle $k-d \cdot g$ is equal to the angle $c$-d.e, for they are opposite angles. Therefore, the angle $l-b \cdot h$ is equal to the angle $k-d-g$. The angle $l \cdot b \cdot h$ is at the center of retinal curvature and is measured by the are $l-h$, and the angle $k \cdot d \cdot g$ is at the center of the retinal curve and is measured by the are $k-g$. The arc $l-h$ must be equal to the are $k-g$, therefore $l$ is related to $h$ as $k$ is related to $g$. The same truth is shown as to retinal points $j$ in the one eye and $f$ in the other eye. And this would be true however much the radius of curvature of the one eye might differ from the radius of curvature of the other eye. The similarity of location of corresponding retinal points is in degrees of arc and not in millimeters. What has been proved of $l$ and $k$ and of $j$ and $f$ as corresponding retinal points, could be proved of all corresponding retinal points lying in the horizontal retinal meridians. Using the same figure, the proof can be applied to points lying in the vertical meridians. With the two eyes fixed, rotate the plane of the circle on the chord $b-d$ as an axis, and thus generate a surface of binocular single vision. As the rotation is effected what were the visual axes $(h-b-c$ and $g-d-c)$ become indirect visual lines, the retinal ends of which are made to move in the vertical retinal meridians. Elevating this plane in front 5 degrees depresses it behind 5 degrees; elevating it in front 10 degrees depresses it behind 10 degrees, and so on for any extent of rotation. The retinal end of the visual line $h-b-c$ and the retinal end of the visual line $g-d-c$ are made to describe arcs of equal degree. What is true of corresponding retinal points in the two horizontal and the two vertical retinal meridians must be true of corresponding retinal points in any two oblique retinal meridians that are similarly related to the vertical and horizontal meridians. Therefore, I conclude that Helmholtz taught the truth concerning corresponding retinal points. Correct as to corresponding retinal points, Helmholtz was in error concerning the normal relationship of the vertical retinal meridians to each other. He should have known that, in accordance with the supreme law of corresponding retinal points, the vertical axes of the eyes, which lie in the planes of the vertical retinal meridians, must be kept parallel with each other, except in cases of non-symmetrical oblique astigmatism. Except for the oblique muscles, the vertical axes of the eyes would not always be correctly related. The law relating to the oblique muscles is that they shall compel the vertical axes of the eyes to remain parallel with the median plane of the head, regardless of the position of the point of fixation in space.

Dr. George T. Stevens-I thi..k that every one who has been a student of physiologic opties recognizes the diagram which has just been thrown on the screen by Dr. Savage as the familiar diagram of Müller. It has figured in text-books for many years. As a practical horopter it was and is an impossibility. It is hardly worth while to discuss the merits of Miiller's horopter, which was rejected by experts long ago. Nor has Helmholtz' horopter been any more satisfactory. Dr. Savage has told us that there is no torsion in the chang ing relations of the eyes except such as results from defects. If this were true, if there are no physiologic torsions, we must abandon the law of Listing. That law has stood for many years and is likely to remain for still many more years. According to that law, in the example which I have chosen, with a depression of 35 degrees and a lateral angle of 5 degrees each eye, there must be torsion of 3 degrees or there will not be single vision. We shall never abolish Listing's law nor shall we find the eyes unchanged in their meridianal relations by their various adjustments. Dr. Savage tells us that he some time since pointed out that the doctrine of Helmholtz relating to the meridians was incorrect. That was not difficult, since I had demonstrated the fact clearly, but it had not been demonstrated until the clinoscope was introduced, when immediately the error was clearly shown.

\section{DENGUE IN THE ISTHMIAN CANAL ZONE.} INCLUDING A REPORT ON THE LABORATORY FINDINGS.* DUDLEY NEWCOMB CARPENTER, M.D. Surgeon U. S. Navy. AND

RICHARD LIGHTBURN SUTTON, M.D. Assistant Surgeon, U. S. Navy.

Of the several tropical diseases whose etiology is yet in question, dengue undoubtedly stands at the head of the list in importance. It has long been considered an infectious disease, as the writings of Ornstein, ${ }^{1}$ Eulenberg, ${ }^{2}$ Graham $^{3}$ and Hirsch ${ }^{4}$ attest, while we find Leichenstein, ${ }^{5}$ Malie, $^{6}$ von Duringe ${ }^{7}$ and a half dozen others presenting almost indisputable evidence that it is contagious, in the technical sense of the word, as well.

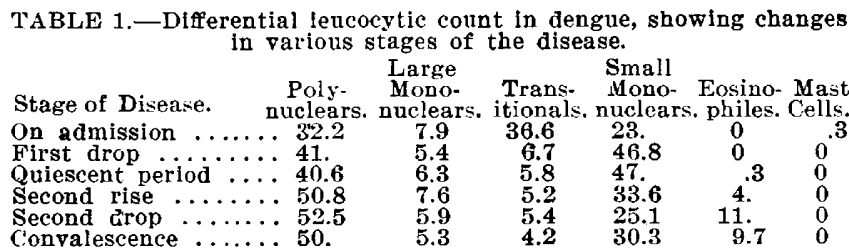

McLaughlin ${ }^{8}$ of Texas, in an exhaustive series of studies begun as far back as 1885, undoubtedly establishes the clearest claim to the discovery of the causative agent, but in spite of his clear and concise description of the method of procedure employed, very few investigators have been ablc to verify the presence in dengue blood of the micrococcus which he found in such large numbers.

Our lack of exact knowledge concerning the morbid anatomy of this infection is principally due to the extremely low mortality, the death rate being 1 per cent. (Hare) or less.

TABLE 2.-Differential leucocytic count in dengue, showing changes
in various stages of the disease.
Large

Consequently we are forced to depend, in a great measure, on examinations of the blood and excreta for information concerning the pathologic changes which are occurring.

During the past spring and summer, while stationed on the Isthmus of Panama, it was our good fortune to be brought into intimate contact with some 200 cases of the disease, and, although no startling discoveries were made, the laboratory findings may serve to direct some future investigator toward the true cause.

* A preliminary report appeared in The Jorrac, Dec. 17, 1904 p. 1869.

1. Zur Dengue, Frage, Deutsche med. Wochenschrift, 1890

2. Realencyklopedie, 1886 , v.

3. Journal of Tropical Medicine, July, 1903.

4. Chrysochoos in Smyrna.

5. Nothnagel's Specielle Pathologie und Theraple, No. 4, vol. 1.

6. Diction Encyclop. des Sclences Medicale.

7. Monatschrift für praktische Dermatologie, 1890.

8. New Orleans Med. and Surg. Jour., 1885, n. s., xiii ; THE Jounnal A. M. A., 1886, vi ; Boston Med. and Surg. Jour., 1886 cxiv: Texas Med. News, 1895-6. 